\title{
Collocation Approximation to Eigenvalues of an Ordinary Differential Equation: The Principle of the Thing
}

\author{
By Carl de Boor and Blair Swartz*
}

\begin{abstract}
It is shown that simple eigenvalues of an $m$ th order ordinary differential equation are approximated within $O\left(|\Delta|^{2 k}\right)$ by collocation at Gauss points with piecewise polynomial functions of degree $<m+k$ on a mesh $\Delta$. The same rate is achieved by certain averages in case the eigenvalue is not simple. The argument relies on an extension and simplification of Osborn's recent results concerning the approximation of eigenvalues of compact linear maps.
\end{abstract}

0. Introduction. The eigenvalue problem we consider is of the form

$$
(M x)(t)=\lambda(N x)(t) \text { for } t \in[0,1], \beta_{i} x=0, i=1, \ldots, m,
$$

where $(\lambda, x) \in \mathbf{C} \times C^{(m)}[0,1]$ is being sought and

$$
\begin{aligned}
& (M x)(t):=\left(D^{m} x\right)(t)+\sum_{i<m} a_{i}(t)\left(D^{i} x\right)(t), \\
& (N x)(t):=\sum_{i<m} b_{i}(t)\left(D^{i} x\right)(t),
\end{aligned}
$$

with $\left(\beta_{i}\right)_{1}^{m}$ a sequence of linear functionals on $C^{(m-1)}[0,1]$. For simplicity, we assume the $\beta_{i}$ to be supported on 0 and/or 1 only; see [4] for a discussion of more general side conditions.

We approximate this problem by collocation, as follows. We choose a (strict) partition $\Delta:=\left(t_{r}\right)_{0}^{l}$ of $[0,1]$,

$$
0=t_{0}<\cdots<t_{l}=1,
$$

and, based on it, a sequence $\left(\tau_{i}\right)_{1}^{k l}$ of collocation points, $k$ to each interval $\left[t_{r}, t_{r+1}\right]$,

$$
\tau_{k r+i}:=\left(t_{r}+t_{r+1}+\rho_{i} \Delta t_{r}\right) / 2, \quad i=1, \ldots, k,
$$

with $\left(\rho_{i}\right)_{1}^{k}$ a fixed sequence of points in the "standard" interval $\left[-1^{+}, 1^{-}\right]$. Then we seek $\lambda_{\Delta} \in \mathbf{C}$ and $x_{\Delta} \in \mathbf{P}_{k+m, \Delta}^{m}:=\mathbf{P}_{k+m, \Delta} \cap C^{(m-1)}[0,1]$ satisfying

$\left(1_{\Delta}\right)\left(M x_{\Delta}\right)\left(\tau_{i}\right)=\lambda_{\Delta}\left(N x_{\Delta}\right)\left(\tau_{i}\right), \quad i=1, \ldots, k l, \beta_{i} x_{\Delta}=0, i=1, \ldots, m$.

Received March 12, 1979; revised October 12, 1979.

1980 Mathematics Subject Classification. Primary 65L 15, 65J05.

Key words and phrases. Eigenvalues, compact linear map, ordinary differential equation, collocation, piecewise polynomial, superconvergence.

* Sponsored by the United States Army under Contract No. DAAG29-75-C-0024 and by the United States Department of Energy under Contract No.W-7405-Eng. 36. 
Here, $\mathbf{P}_{r, \Delta}$ consists of all functions on $[0,1]$ which, on each interval $\left[t_{j}^{+}, t_{j+1}^{-}\right]$, coincide with some polynomial of order $r$, or, of degree $<r$.

In this paper, we bound the difference between the eigenelement $(\lambda, x)$ and its collocation approximation $\left(\lambda_{\Delta}, x_{\Delta}\right)$, as a function of the mesh size $|\Delta|:=$ $\max _{i} \Delta t_{i}$, the ascent of $\lambda$, and the choice of the collocation pattern $\left(\rho_{i}\right)_{i}^{k}$. In particular, we show, as a consequence of Theorem 3.1, that

$$
\lambda_{\Delta}=\lambda+O\left(|\Delta|^{2 k}\right)
$$

in case $\lambda$ has ascent 1 and $\left(\rho_{i}\right)_{1}^{k}$ are the Gauss-Legendre points. We also show that it is not possible to obtain this result as an application of Osborn's [11] general theory. Further, we show that (4) holds also when $\lambda$ has ascent greater than 1 , provided $\lambda_{\Delta}$ is replaced by an average of certain nearby approximate eigenvalues.

Here is an outline of the paper. In Section 1, we identify collocation as a particular projection method applied to the eigenvalue problem

$$
\lambda T y=y
$$

for a certain compact linear map $T$ on an appropriate Banach space. In Section 2, we apply Osborn's nice results to this projection method, indicate that one cannot obtain (4) in that way, but defer a proof of this claim to Section 4. We then modify Osborn's analysis appropriately in order to relate, in Theorem 2.2 , the error $\lambda-\lambda_{\Delta}$ to numbers of the form

$$
\varphi *\left(T-T_{\Delta}\right) \varphi_{\Delta}
$$

with $\varphi_{\Delta}$ an approximate (generalized) eigenvector and $\varphi^{*}$ a (generalized) eigenvector of the adjoint problem. In Section 3, these numbers are shown to be $O\left(|\Delta|^{2 k}\right)$ for collocation at Gauss points, which leads to (4). Section 3 also contains a short discussion of related results in the literature.

We have deferred discussion of numerical examples to a companion paper [3] We found only one real example of an ordinary differential equation with an eigenvalue of ascent greater than one in the literature. Yet, existing theory (and the theory developed in the present paper) give convergence results which strongly depend on the ascent. The three examples we give do show that the proven convergence rates cannot be improved. We also offer an explanation of sorts for the curious way in which approximate eigenvalues have, in some examples, been observed to converge to an eigenvalue of ascent greater than one.

In the process of writing the present paper, we have reexamined the, at times, convoluted arguments in de Boor and Swartz [2] for the superconvergence of Gauss point collocation approximations at knots. This has led us to a whole family of projectors onto $\mathbf{P}_{m+k, \Delta}^{m}$, of which interpolation at the Gauss collocation points is only one example, which all lead to $O\left(|\Delta|^{2 k}\right)$ approximations at knots and therefore give $O\left(|\Delta|^{2 k}\right)$ approximations to simple eigenvalues. We discuss these ideas in [4], where we also give an alternative proof of Lemma 3.1. 
1. Translation to an Abstract Setup. We would like to discuss collocation in the setting of Osborn [11], Vainikko [12] et al., i.e., as a problem of approximating the eigenelements of a compact map $T$ on some Banach space $Y$, and therefore assume that 0 is not an eigenvalue of $(0.1)$ and that the coefficients of $M$ are continuous (later, we will assume them to be quite smooth). Then, $M^{-1}$ exists as an integral operator on $Y:=\mathbf{L}_{p}[0,1], 1 \leqslant p \leqslant \infty$,

$$
\left(M^{-1} y\right)(t)=\int_{0}^{1} G(t, s) y(s) d s
$$

with $G$ Green's function for the problem

$$
(M x)(t)=y(t) \text { for } t \in[0,1], \beta_{i} x=0, i=1, \ldots, m .
$$

Further, $(\lambda, x)$ is an eigenelement of $(0.1)$ if and only if $(1 / \lambda, M x)$ is an eigenelement of the compact linear map

$$
T:=N M^{-1}
$$

on $Y$. Note that $T$ is given as an integral operator with a piecewise continuous kernel $T$,

$$
T(t, s):=\sum_{i=0}^{m-1} b_{i}(t)(\partial / \partial t)^{i} G(t, s),
$$

therefore compact as a map from $Y$ into $C$. In fact, $T$ maps $Y$-bounded sets to uniformly Lipschitz continuous sets.

Such a change transforms $\left(0.1_{\Delta}\right)$ into the problem of finding $\left(\lambda_{\Delta}, y_{\Delta}\right) \in \mathrm{C} \times V_{\Delta}$ such that

$$
y_{\Delta}\left(\tau_{i}\right)=\lambda_{\Delta}\left(T y_{\Delta}\right)\left(\tau_{i}\right), \quad i=1, \ldots, k l
$$

with

$$
V_{\Delta}:=\left\{M z: z \in \mathbf{P}_{m+k, \Delta}^{m}, \beta_{i} z=0, i=1, \ldots, m\right\}
$$

We convert this into an ordinary eigenvalue problem involving some operator $T_{\Delta}$ on $Y$ by introducing the linear projector $P_{\Delta}$, which carries $C_{\Delta}[0,1]\left(:=C\left[t_{0}, t_{1}\right] \times \cdots\right.$ $\left.\times C\left[t_{l-1}, t_{l}\right]\right)$ onto $V_{\Delta}$ by associating with each $y \in C_{\Delta}[0,1]$ the unique element $P_{\Delta} y \in V_{\Delta}$ for which

$$
\left(P_{\Delta} y\right)\left(\tau_{i}\right)=y\left(\tau_{i}\right), \quad i=1, \ldots, k l .
$$

Then the two conditions: $f \in V_{\Delta}$ and $f\left(\tau_{i}\right)=g\left(\tau_{i}\right), i=1, \ldots, k l$, are equivalent to the one condition: $f=P_{\Delta} g$. The approximate eigenvalue problem $\left(0.1_{\Delta}\right)$ is therefore equivalent to the problem of finding $\left(\lambda_{\Delta}, y_{\Delta}\right) \in \mathrm{C} \times Y$ such that

$$
y_{\Delta}=\lambda_{\Delta} T_{\Delta} y_{\Delta}
$$

with

$$
T_{\Delta}:=P_{\Delta} T
$$


Of course, it is not clear a priori that $P_{\Delta}$ is even defined. But note that, by definition, $P_{\Delta} y$ is the unique element in $V_{\Delta}$, i.e., of the form $M x_{\Delta}$ with $x_{\Delta} \in$ $\mathbf{P}_{k+m, \Delta}^{m}$ and $\beta_{i} x_{\Delta}=0, i=1, \ldots, m$, which agrees with $y$ at the $\tau_{i}$ 's. According to Theorem 3.1 of de Boor and Swartz [2], there exists a positive const ${ }_{M}$ depending on $M($ and $\rho$ ) so that, for all partitions $\Delta$ with

$$
|\Delta|:=\max _{i} \Delta t_{i} \leqslant \text { const }_{M}
$$

and all $y \in C_{\Delta}[0,1]$, the collocation equation

$$
\left(M x_{\Delta}\right)\left(\tau_{i}\right)=y\left(\tau_{i}\right), \quad i=1, \ldots, k l, \beta_{i} x_{\Delta}=0, i=1, \ldots, m,
$$

has exactly one solution, $x_{\Delta}$, in $\mathbf{P}_{k+m, \Delta}^{m}$. (To be precise, de Boor and Swartz [2] assume additionally that $\left(\beta_{i}\right)_{1}^{m}$ is linearly independent over $\mathbf{P}_{m}$, but this assumption was shown to be superfluous by Wittenbrink [16]. Also, both papers only consider real valued functions, but the extension to complex valued functions is trivial. Wittenbrink's formulation would also appear to be appropriate for problems in which the $\beta_{i}$ also depend on $\lambda$.) This insures that $P_{\Delta}$ is defined for all $\Delta$ with $|\Delta|$ sufficiently small, and allows us to conclude from [2] that $P_{\Delta}$ converges to the identity pointwise on $C[0,1]$.

In fact, if $\left(\beta_{i}\right)_{1}^{m}$ is linearly independent over $\mathbf{P}_{m}=\operatorname{ker} D^{m}$, then $P_{\Delta}=$ $\left(D^{m} M^{-1}\right)^{-1} P\left(D^{m} M^{-1}\right)$, with $P$ the linear projector taking $D^{m} x$ to $D^{m} x_{\Delta}$ and shown in [2] to be bounded on $C[0,1]$ independently of $\Delta$; hence $P_{\Delta}$ is then bounded on $C[0,1]$ independently of $\Delta$. In the more general case, an argument like Wittenbrink's (see [16, proof of Theorem 2]) shows that

$$
\left\|\left(1-P_{\Delta}\right) y\right\|_{\infty} \leqslant \text { const }\left\|\left(1-Q_{\Delta}\right) D^{m} M^{-1} y\right\|_{\infty}
$$

for some const independent of $\Delta$ and with $Q_{\Delta}$ interpolation from $\mathbf{P}_{k, \Delta}$ at the collocation points $\left(\tau_{i}\right)_{1}^{k l}$.

In conclusion, not only is $P_{\Delta}$ defined for all small $|\Delta|$, but, since $T$ maps $Y$ compactly into $C$, we have $T_{\Delta}=P_{\Delta} T$ defined and uniformly convergent to $T$ as $|\Delta| \rightarrow 0$.

2. A General Result. In this section, we recall Osborn's results and then rephrase his arguments to obtain a simple yet useful formulation of the error in eigenvalue approximations by projection (and other) methods. This formulation makes it evident (as Osborn's or Vainikko's does not) why eigenvalue approximations by collocation are of such high order.

We recall from Osborn [11] (or from a standard reference such as Kato [8]) that, associated with each nonzero eigenvalue $\mu$ of a compact linear map $T$ on the Banach space $Y$ is the invariant subspace

$$
S:=\operatorname{ker}(\mu-T)^{\alpha}
$$

i.e., the kernel or nullspace of the linear map $(\mu-T)^{\alpha}$, where $\alpha$, the ascent or rank of $\mu$, is the smallest integer for which $\operatorname{ker}(\mu-T)^{\alpha}=\operatorname{ker}(\mu-T)^{\alpha+1} . S$ is finite dimensional, say

$$
a:=\operatorname{dim} S=: \text { algebraic multiplicity of } \mu \text {, }
$$


while the geometric multiplicity of $\mu$ is the number $g:=\operatorname{dim} \operatorname{ker}(\mu-T)$. The elements of $\operatorname{ker}(\mu-T) \backslash\{0\}$ are the eigenvectors of $T$, while those of $S \backslash \operatorname{ker}(\mu-T)$ are called root vectors or generalized eigenvectors. $S$ is the range of the linear projector $E$ given, e.g., by the formula

$$
E:=\frac{1}{2 \pi i} \int_{\Gamma}(z-T)^{-1} d z
$$

with $\Gamma$ any circle in the complement of the spectrum of $T$ and enclosing in its interior $\mu$ but no other eigenvalue of $T$. The linear projector $E$ can also be written in the form

$$
E y=\sum_{i=1}^{a}\left(\varphi_{i}^{*} y\right) \varphi_{i}
$$

with $\left(\varphi_{i}\right)_{1}^{a}$ any basis for $S$ and $\left(\varphi_{i}^{*}\right)_{1}^{a}$ the corresponding dual basis in

$$
S^{*}:=\operatorname{ker}\left(\mu-T^{*}\right)^{\alpha} \text {, }
$$

i.e., $\varphi_{i}^{*} \in S^{*}$, all $i$, and $\varphi_{i}^{*} \varphi_{j}=\delta_{i j}$, all $i, j$. Here, $T^{*}$ is the (Banach)-adjoint or $d u a l$ of $T$, i.e., the linear map on the topological dual $Y^{*}$ of $Y$ which carries $y^{*} \in Y^{*}$ to $y^{*} T \in Y^{*}$. In particular, $S^{*}$ is the invariant subspace of $T^{*}$ corresponding to $\mu$ and has also dimension $a$.

In terms of this notation, Osborn [11] establishes (among others) the following facts.

THEOREM 2.1 (OSBORN). Let $\left(T_{\Delta}\right)$ be a sequence or net of compact linear maps on the Banach space $Y$ converging uniformly to the linear map $T$ as $|\Delta| \rightarrow 0$ and let $\mu$ be a (nonzero) eigenvalue of $T$. Then, for all small $|\Delta|$,

$$
E_{\Delta}:=\frac{1}{2 \pi i} \int_{\Gamma}\left(z-T_{\Delta}\right)^{-1} d z
$$

is defined; its range $S_{\Delta}$ has dimension a and satisfies

$$
\operatorname{gap}\left(S, S_{\Delta}\right) \leqslant \text { const }\left\|\left.\left(T-T_{\Delta}\right)\right|_{S}\right\|
$$

with

$$
\operatorname{gap}(U, V):=\max \left\{\sup _{x \in U} \frac{\operatorname{dist}(x, V)}{\|x\|}, \sup _{x \in V} \frac{\operatorname{dist}(x, U)}{\|x\|}\right\} .
$$

Further, if $\mu_{\Delta}$ is one of the eigenvalues of $T_{\Delta}$ enclosed by $\Gamma$, then

$$
\left|\mu-\mu_{\Delta}\right|^{\alpha} \leqslant \text { const }\left(\left\|\left.\left(T-T_{\Delta}\right)\right|_{S}\right\| \|\left.\left(T^{*}-T_{\Delta}^{*}\right)\right|_{S * \|}+\sum_{i, j=1}^{a}\left|\varphi_{i}^{*}\left(T-T_{\Delta}\right) \varphi_{j}\right|\right) \text {. }
$$

However, the average

$$
\bar{\mu}_{\Delta}:=\left(\mu_{1}+\cdots+\mu_{a}\right) / a,
$$

comprised of the a eigenvalues of $T_{\Delta}$ (counting algebraic multiplicities) enclosed by $\Gamma$, 
admits the closer estimate

(8) $\left|\mu-\bar{\mu}_{\Delta}\right| \leqslant$ const $\left\{\left\|\left.\left(T-T_{\Delta}\right)\right|_{S}\right\|\left\|\left.\left(T^{*}-T_{\Delta}^{*}\right)\right|_{S^{*}}\right\|+\sum_{i=1}^{a}\left|\varphi_{i}^{*}\left(T-T_{\Delta}\right) \varphi_{i}\right|\right\}$.

This is a powerful theorem, and Osborn makes good use of it in a variety of applications described at the end of his paper, mostly to Galerkin approximations. But the theorem does not suffice to explain the high accuracy of eigenvalue approximations obtained by collocation. The chief difficulty lies in the fact that in collocation (as translated, in the preceding section, into this abstract setup) $T_{\Delta}^{*}$ does not approximate $T^{*}$ sufficiently well; see Section 4 .

We obtain more direct control by comparing matrix representations for $\left.T\right|_{S}$ and $\left.T_{\Delta}\right|_{s_{\Delta}}$, as is done, in effect, in Osborn [11] to get the estimates (7) and (8), and is done, quite explicitly, in Atkinson [1] and in Kreiss [9]; but we do it a little bit differently.

Let $J$ be the matrix representation of $\left.T\right|_{S}$ with respect to the basis $\left(\varphi_{i}\right)_{1}^{a}$ for $S$. Since $\operatorname{gap}\left(S, S_{\Delta}\right) \rightarrow 0,\left.E\right|_{S_{\Delta}}$ is 1-1 for all small $|\Delta|$, and then there is a corresponding basis $\left(\varphi_{i, \Delta}\right)_{1}^{a}$ for $S_{\Delta}$ with

$$
E \varphi_{i, \Delta}=\varphi_{i}, \quad i=1, \ldots, a .
$$

Since, by (3), $\varphi_{j}^{*}=\varphi_{j}^{*} E$, we see that

$$
\varphi_{i}^{*} \varphi_{j, \Delta}=\varphi_{i}^{*} E \varphi_{j, \Delta}=\varphi_{i}^{*} \varphi_{j}=\delta_{i j}
$$

hence $y=\Sigma_{i}\left(\varphi_{i}^{*} y\right) \varphi_{i, \Delta}$ for all $y \in S_{\Delta}$, thus the matrix representation $J_{\Delta}$ for $\left.T_{\Delta}\right|_{s_{\Delta}}$ with respect to $\left(\varphi_{i, \Delta}\right)_{1}^{a}$ has $(i, j)$-entry

$$
\left(J_{\Delta}\right)_{i j}=\varphi_{i}^{*} T_{\Delta} \varphi_{j, \Delta}, \quad i, j=1, \ldots, a .
$$

But the entries of $J$ are given by a similar expression. We have

$$
(J)_{i j}=\varphi_{i}^{*} T \varphi_{j}=\varphi_{i}^{*} T E \varphi_{j, \Delta}=\varphi_{i}^{*} E T \varphi_{j, \Delta}=\varphi_{i}^{*} T \varphi_{j, \Delta}, \quad i, j=1, \ldots, a,
$$

using the fact that $T$ commutes with its spectral projector $E$. Consequently,

$$
\left\|J-J_{\Delta}\right\| \leqslant \text { const }_{\|\cdot\|} \max _{i, j}\left|\varphi_{i}^{*}\left(T-T_{\Delta}\right) \varphi_{j, \Delta}\right| .
$$

We gather these facts into a theorem.

THEOREM 2.2. With the assumptions and notations of Theorem 2.1 , let $J$ be the matrix representation of $\left.T\right|_{S}$ with respect to some basis $\left(\varphi_{i}\right)_{1}^{a}$ of $S$. Then, $\left.T_{\Delta}\right|_{S_{\Delta}}$ is similar to a matrix $J_{\Delta}$ close to $J$ in the sense that

$$
\left(J-J_{\Delta}\right)_{i j}=\varphi_{i}^{*}\left(T-T_{\Delta}\right) \varphi_{j, \Delta}, \quad i, j=1, \ldots, a,
$$

with $\left(\varphi_{j, \Delta}\right)_{1}^{a}$ the basis for $S_{\Delta}$ for which $E \varphi_{j, \Delta}=\varphi_{j}$, all $j$, and $\left(\varphi_{i}^{*}\right)_{1}^{a}$ the basis for $S^{*}$ dual to $\left(\varphi_{i}\right)_{1}^{a}$ (and to $\left.\left(\varphi_{i, \Delta}\right)_{1}^{a}\right)$.

It follows that the eigenvalues of $T_{\Delta}$ close to $\mu$ are those of the matrix $J_{\Delta}$ (including algebraic multiplicity and structure) and, since $J$ has $\mu$ as an eigenvalue in just 
the same way as $T$ does (including algebraic multiplicity and structure), the approximation properties of the process can be read off from standard perturbation arguments which compare the eigenvalues of a matrix $J$ with those of a perturbation $J_{\Delta}$, e.g., from Wilkinson [13], in the manner practiced by Atkinson [1] and Kreiss [9].

In particular,

$$
\left|\mu-\mu_{\Delta}\right| \leqslant \text { const }\left\|J-J_{\Delta}\right\|^{1 / \alpha}
$$

for any of the $a$ eigenvalues $\mu_{\Delta}$ of $J_{\Delta}$, while

$$
\left|\mu-\left(\operatorname{trace} J_{\Delta}\right) / a\right|=\left|\operatorname{trace}\left(J-J_{\Delta}\right)\right| / a \leqslant \text { const }\left\|J-J_{\Delta}\right\|,
$$

and also

$$
\left|1 / \mu-\left(\operatorname{trace} J_{\Delta}^{-1}\right) / a\right| \leqslant \text { const }\left\|J-J_{\Delta}\right\| \text {. }
$$

In fact, with $\mu_{1}, \ldots, \mu_{a}$ the eigenvalues of $J_{\Delta}$ counting algebraic multiplicities, and $\sigma_{0}, \ldots, \sigma_{a}$ the elementary symmetric functions in $a$ variables, we see, by comparing coefficients of the characteristic polynomials of $J$ and $J_{\Delta}$, that

$$
\sigma_{j}\left(\mu_{1}, \ldots, \mu_{a}\right)=\sigma_{j}(\mu, \ldots, \mu)+O\left(\left\|J-J_{\Delta}\right\|\right), \text { all } j .
$$

Finally, with $g$ the geometric multiplicity of $\mu$, there exists an eigenvalue $\mu_{\Delta}$ of $J_{\Delta}$ (and $T_{\Delta}$ ) so that

$$
\mu=\mu_{\Delta}+O\left(\left\|J-J_{\Delta}\right\|^{g / a}\right) .
$$

This is a better rate than (12) except when all Jordan blocks of $J$ have the same size.

The estimate (16) appears in Wilkinson [13, p. 81], while (13), (14) and (15) are obvious. The proof of (12) (via Gershgorin's circle theorem) is left to the reader in Wilkinson [13, pp. 80-81]. Atkinson [1] gives, so he says, a shorter proof. We record our own version, for the record.

Since $(\mu-J)^{\alpha}=0$,

$$
\left|\mu-\mu_{\Delta}\right|^{\alpha} \leqslant\left\|\left(\mu-J_{\Delta}\right)^{\alpha}\right\|=\left\|\left(\mu-J_{\Delta}\right)^{\alpha}-(\mu-J)^{\alpha}\right\| \leqslant \text { const }_{\|J\|,\left\|J_{\Delta}\right\|}\left\|J-J_{\Delta}\right\|,
$$

using the fact that the map $A \mapsto A^{\alpha}$ is locally Lipschitz continuous. (Indeed, $A^{\alpha}-B^{\alpha}$ $=\Sigma_{j=0}^{\alpha-1} A^{\alpha-j-1}(A-B) B^{j}$, hence $\left.\left\|A^{\alpha}-B^{\alpha}\right\| \leqslant\left[\sum_{j=0}^{\alpha-1}\|A\|^{\alpha-j-1}\|B\|^{j}\right]\|A-B\|.\right)$ This proves (12), given that $J_{\Delta} \rightarrow J$.

Note that we recover (7)-(8) of Osborn's theorem directly from (12)-(13) because of (10) and since

$$
\begin{aligned}
\left(J-J_{\Delta}\right)_{i j} & =\varphi_{i}^{*}\left(T-T_{\Delta}\right)\left(\left.E\right|_{S_{\Delta}}\right)^{-1} \varphi_{j} \\
& =\varphi_{i}^{*}\left(T-T_{\Delta}\right)\left[\left(\left.E\right|_{S_{\Delta}}\right)^{-1} \varphi_{j}-\varphi_{j}\right]+\varphi_{i}^{*}\left(T-T_{\Delta}\right) \varphi_{j}
\end{aligned}
$$

while

$$
\begin{aligned}
\left\|\left(\left.E\right|_{S_{\Delta}}\right)^{-1} \varphi-\varphi\right\| & \leqslant\|1-E\| \operatorname{dist}\left(\left(\left.E\right|_{S_{\Delta}}\right)^{-1} \varphi, \operatorname{ran} E\right) \\
& \leqslant\|1-E\| \text { const }\left\|\left.\left(T-T_{\Delta}\right)\right|_{S}\right\|\|\varphi\|,
\end{aligned}
$$


the last by (5) and because $\left\|\left(\left.E\right|_{S_{\Delta}}\right)^{-1} \varphi\right\| \leqslant$ const $\|\varphi\|$ for all sufficiently small $|\Delta|$. Here we have used the facts that $\left(\left.E\right|_{S_{\Delta}}\right)^{-1} \varphi-\varphi=(1-E)\left(\left.E\right|_{S_{\Delta}}\right)^{-1} \varphi$, and that $E$ is a linear projector onto $S$, hence $\|(1-E) v\| \leqslant\|1-E\| \operatorname{dist}(v, S)$ while, for $v \in S_{\Delta}$, $\operatorname{dist}(v, S) \leqslant \operatorname{gap}\left(S, S_{\Delta}\right)\|v\|$.

It remains, for any particular approximation sequence or net $\left(T_{\Delta}\right)$, to estimate $\left\|J-J_{\Delta}\right\|$, i.e., to estimate expressions of the form

$$
\varphi^{*}\left(T-T_{\Delta}\right)\left(\left.E\right|_{S_{\Delta}}\right)^{-1} \varphi
$$

with $\varphi \in S, \varphi^{*} \in S^{*}$. The estimate

$$
\left\|J-J_{\Delta}\right\| \leqslant \mathrm{const}\left\|\left.\left(T-T_{\Delta}\right)\right|_{S}\right\|
$$

is, of course, immediate from (17) and (18) and provides, in conjunction with (12)(16), first assurance that the eigenvalues of $T$ are, indeed, approximated well by those of $T_{\Delta}$. It also makes the important point that the error in the eigenvalue approximation achieved by a particular discretization method is at least of the same order as the error in the corresponding discrete solution of the corresponding nonsingular equation. But this estimate ignores the fact that the linear functional $\varphi^{*}$ is being applied to a residual error, i.e., to an element of the form $\left(T-T_{\Delta}\right) y$, a fact that, at times, leads to sharper estimates. For example, assume that $T_{\Delta}$ is obtained from $T$ by projection, i.e., $T_{\Delta}=P_{\Delta} T$ for some projector $P_{\Delta}$ defined on some subspace $Y_{\Delta}$ of $Y$ in the range of $T$. Then $P_{\Delta}^{*}$ is defined on the superspace $Y_{\Delta}^{*}$ of $Y^{*}$ and, as in (17) and (18),

$$
\begin{aligned}
\left|\varphi^{*}\left(T-T_{\Delta}\right)\left(\left.E\right|_{S_{\Delta}}\right)^{-1} \varphi\right| \\
\quad=\left|\left[\varphi^{*}\left(1-P_{\Delta}\right)\right] T\left[\left(\left.E\right|_{S_{\Delta}}\right)^{-1} \varphi-\varphi\right]+\left[\varphi^{*}\left(1-P_{\Delta}\right)\right]\left(1-P_{\Delta}\right) T \varphi\right| \\
\quad \leqslant \operatorname{const}\left\|\left.\left(1-P_{\Delta}\right)\right|_{S}\right\|\left\|\left.\left(1-P_{\Delta}\right)^{*}\right|_{S}\right\|\|\varphi\|\left\|\varphi^{*}\right\|
\end{aligned}
$$

(using the facts that $\left(1-P_{\Delta}\right)^{2}=\left(1-P_{\Delta}\right)$ and that $\left.T S \subseteq S\right)$. This provides an indication of the potential "double accuracy" in the eigenvalue approximation by such methods, as first proved by Vainikko [12], and also established in this generality by Osborn [11].

This potential "double accuracy" or, at least, higher order of convergence (as compared with (20)) comes from the extra factor $\left\|\left.\left(1-P_{\Delta}\right)^{*}\right|_{S^{*}}\right\|$, whose rate of convergence to zero (as $|\Delta| \rightarrow 0$ ) is usually inferred from the inequality

$$
\left\|\left.\left(1-P_{\Delta}\right)^{*}\right|_{S}\right\| \leqslant\left\|1-P_{\Delta}\right\| \sup _{\varphi^{*} \in S^{*}} \operatorname{dist}\left(\varphi^{*}, \operatorname{ran} P_{\Delta}^{*}\right) /\left\|\varphi^{*}\right\|
$$

For example, in the analysis of Galerkin's method, or the least-squares method, $\varphi^{*} \in S^{*}$ is given by integration,

$$
\varphi^{*} f=\int \varphi^{*}(s) f(s) d s
$$

for some smooth function $\varphi^{*}$, while $\operatorname{ran} P_{\Delta}^{*}$, i.e., the interpolation conditions for $P_{\Delta}$, consists of integration against any $g$ in the span of certain piecewise smooth functions $K_{j}$. 
Explicitly, then

$$
\operatorname{dist}\left(\varphi^{*}, \operatorname{ran} P_{\Delta}^{*}\right)=\inf _{\gamma} \sup _{f \in Y}\left|\int \varphi^{*}(s) f(s) d s-\int \sum_{i} \gamma_{i} K_{i}(s) f(s) d s\right| /\|f\|,
$$

with $\operatorname{span}\left(K_{i}\right)$ akin to $\mathbf{P}_{k, \Delta}$ in approximation power. This allows the conclusion that

$$
\left\|\left.\left(1-P_{\Delta}\right)^{*}\right|_{S} *\right\|=O\left(|\Delta|^{k}\right)
$$

for these methods, which, together with the more obvious fact that $\left\|\left.\left(1-P_{\Delta}\right)\right|_{S}\right\|=$ $O\left(|\Delta|^{k}\right)$, gives the sought-for "double accuracy" for such methods.

For collocation, though, $\operatorname{dist}\left(\varphi^{*}, \operatorname{ran} P_{\Delta}^{*}\right)$ fails to go to zero with any kind of rapidity, since now

$$
\operatorname{dist}\left(\varphi^{*}, \operatorname{ran} P_{\Delta}^{*}\right)=\inf _{\gamma} \sup _{f \in Y}\left|\int \varphi^{*}(s) f(s) d s-\sum_{i} \gamma_{i} f\left(\tau_{i}\right)\right| \mid\|f\|,
$$

i.e., $\operatorname{ran} P_{\Delta}^{*}$ consists of linear combinations of certain point evaluations. Therefore, (21) in conjunction with the simple bound (22) only implies $O\left(|\Delta|^{k}\right)$ convergence for Gauss point collocation. Even (21) itself, i.e. Osborn's bound (8) only implies $O\left(|\Delta|^{k+\hat{m}}\right)$ convergence for Gauss point collocation, with $\hat{m}$ the difference between the order $m$ of $M$ and the order of $N$; see Section 4 .

Because of this, we proved Theorem 2.2 which relates the error in the eigenvalue approximation not to some operator norms, but only to the value of certain smooth linear functionals on the residual error in a collocation approximation. For such expressions can be shown to be $O\left(|\Delta|^{2 k}\right)$, as is done in the next section.

3. Application to Collocation at Gauss Points. For collocation, as described in the introductory sections 0 and 1 , the quantities $\varphi_{i}^{*}\left(T-T_{\Delta}\right) \varphi_{j, \Delta}$ in the crucial bound (2.10) take the form

$$
\int_{0}^{1} \varphi_{i}^{*}(t)\left(T-T_{\Delta}\right) \varphi_{j, \Delta}(t) d t
$$

and we now intend to show these to be $O\left(|\Delta|^{2 k}\right)$, if the collocation points are chosen as Gauss points, i.e. if $\left(\rho_{i}\right)_{1}^{k}$ consists of the $k$ zeros of the Legendre polynomial of degree $k$. The analysis of collocation at Gauss points in de Boor and Swartz [2] was based on the observation that

$$
\int_{0}^{1} \varphi^{*}(t)\left(T-T_{\Delta}\right) \varphi(t) d t=O\left(|\Delta|^{2 k}\right)
$$

in case $\varphi^{*}$ and $\varphi$ are both smooth. But, since (1) involves $\varphi_{j, \Delta}$ rather than a fixed $\varphi_{j}$, we have to make more explicit just how (2) depends on $\varphi$, and, because of our desire to keep our estimates mesh independent, i.e., dependent only on the number $|\Delta|$, the argument is a bit delicate.

We continue to use the notation and terms of the preceding section. Also, we use the abbreviations

$$
\|\varphi\|_{(r)}:=\sup _{t_{r}<t<t_{r+1}}|\varphi(t)|
$$


and

$$
\|\varphi\|_{s,(r)}:=\sum_{i=0}^{s}\left\|D^{i} \varphi\right\|_{(r)}
$$

Further, we assume that $\left(\rho_{i}\right)_{1}^{k}$ has been chosen so that

$$
\int_{-1}^{1} p(t) \prod_{i=1}^{k}\left(t-\rho_{i}\right) d t=0 \quad \text { for all } p \in \mathbf{P}_{n} .
$$

Finally, we assume that the coefficients of $M$ and $N$ are smooth enough. Specifically, we assume that

$$
a_{i}, b_{i} \in C^{(n+k)}[0,1], \text { all } i
$$

Then, using the abbreviation

$$
X^{(r)}:=\left\{f \in C^{(r-1)}[0,1]: D^{r-1} f \text { abs. cont., } D^{r} f \in X\right\}
$$

with $X$ any of the spaces $\mathrm{L}_{p}[0,1], 1 \leqslant p \leqslant \infty$, or $C[0,1]$, the map $M^{-1}$ carries $X^{(r)}$ into $X^{(r+m)}$, and $N$ maps $X^{(r+m)}$ into $X^{(r+1)}$, for $r<n+k$. In particular, any invariant subspace of $T$ belonging to a nonzero eigenvalue consists of functions in $X^{(n+k)}$, and the same holds for $T^{*}$, the adjoint of $T$. This insures that

$$
S \subseteq C^{(n+k)}, \quad S^{*} \subseteq C^{(n)},
$$

and implies, with (1.7), that

$$
\left\|\left.\left(1-P_{\Delta}\right)\right|_{S}\right\|_{\infty}=O\left(|\Delta|^{k}\right) .
$$

In particular, $\left\|J-J_{\Delta}\right\|=O\left(|\Delta|^{k}\right)$, by (2.20) (using specifically $Y=\mathbf{L}_{\infty}[0,1]$ in the development leading up to it), and (2.12) now gives the results of $R$. Winther [14] for collocation.

We also conclude from (6) and (2.18) (with $\left.Y=\mathrm{L}_{\infty}[0,1]\right)$ that

$$
\left\|\left(\left.E\right|_{S_{\Delta}}\right)^{-1} \varphi-\varphi\right\|_{\infty}=O\left(|\Delta|^{k}\right)\|\varphi\| \quad \text { for } \varphi \in S
$$

LEMma 3.1. There is a constant const depending only on $k$ and $\rho$ so that

$$
\left|\int_{t_{r}}^{t_{r+1} \varphi^{*}\left(T-T_{\Delta}\right) \varphi_{\Delta}}\right| \mid \leqslant \text { const }\left|\Delta t_{r}\right|^{n+k+1}\left\|\varphi^{*}\right\|_{n,(r)}\left\|\left(T-T_{\Delta}\right) \varphi_{\Delta}\right\|_{n+k,(r)} .
$$

Proof. The function $\left(T-T_{\Delta}\right) \varphi_{\Delta}$ vanishes at the $k$ collocation points $\tau_{r k+1}, \ldots, \tau_{r k+k}$ in $\left[t_{r}, t_{r+1}\right]$ and, by assumption (3), the polynomial $\Pi_{s}\left(t-\rho_{r k+s}\right)$ is orthogonal to $\mathbf{P}_{n}$ on $\left[t_{r}, t_{r+1}\right]$. The argument in de Boor and Swartz [2], in the proof of Theorem 4.1 (with $\varphi^{*}$ playing the role of $G(t, \cdot)$ ), therefore establishes the inequality, assuming, of course, that $\varphi^{*} \in \mathrm{L}_{\infty}^{(n)}\left[t_{r}, t_{r+1}\right]$ and $\left(T-T_{\Delta}\right) \varphi_{\Delta} \in$ $\mathbf{L}_{\infty}^{(n+k)}\left[t_{r}, t_{r+1}\right]$.

Next, consider $\left\|\left(T-T_{\Delta}\right) \varphi_{\Delta}\right\|_{n+k,(r)}$ with $\varphi_{\Delta}=\varphi_{j, \Delta}=\left(\left.E\right|_{S_{\Delta}}\right)^{-1} \varphi_{j}$. We would like to bound this number in terms of $\varphi_{j, \Delta}$ and its derivatives on $\left(t_{r}, t_{r+1}\right)$, and this 
seems difficult since $T_{\Delta} f$ at a point depends, offhand, on $f$ on all of $[0,1]$. But

$$
T_{\Delta} \varphi_{j, \Delta}=\sum_{i=1}^{a}\left(J_{\Delta}\right)_{i j} \varphi_{i, \Delta},
$$

since $J_{\Delta}=\left(\varphi_{i}^{*} T_{\Delta} \varphi_{j, \Delta}\right)$ represents $\left.T_{\Delta}\right|_{s_{\Delta}}$ with respect to $\left(\varphi_{i, \Delta}\right)$. Therefore, with

$$
x_{i, \Delta}:=M^{-1} \varphi_{i, \Delta} \in \mathbf{P}_{m+k, \Delta}^{m}, \quad i=1, \ldots, a,
$$

we have

$$
\left(T-T_{\Delta}\right) \varphi_{j, \Delta}=T \varphi_{j, \Delta}-\sum_{i}\left(J_{\Delta}\right)_{i j} \varphi_{i, \Delta}=N x_{j, \Delta}-\sum_{i}\left(J_{\Delta}\right)_{i j} M x_{i, \Delta},
$$

and $J_{\Delta} \rightarrow J$. This shows the derivatives of $\left(T-T_{\Delta}\right) \varphi_{j, \Delta}$ to be expressible in terms of the derivatives of the coefficient functions of $M$ and $N$ (it is only here that we use the full power of our assumption (4)) and those of $x_{i, \Delta} \in \mathbf{P}_{k+m, \Delta}^{m}$, and, as $J_{\Delta} \rightarrow J$ (by (2.20)), it follows that

$$
\left\|\left(T-T_{\Delta}\right) \varphi_{j, \Delta}\right\|_{n+k,(r)} \leqslant \text { const }_{M, N} \max _{i}\left\|x_{i, \Delta}\right\|_{m+k,(r)} .
$$

To complete the analysis, it remains to bound $\left\|x_{i, \Delta}\right\|_{m+k,(r)}$ appropriately.

LEMMA 3.2. For $\varphi \in S$ and $x:=M^{-1} \varphi$, let $x_{\Delta}:=M^{-1} \varphi_{\Delta}$ with $\varphi_{\Delta}:=$ $\left(\left.E\right|_{s_{\Delta}}\right)^{-1} \varphi$. Then, for $s=0, \ldots, m+k$,

$$
\left\|D^{s} x_{\Delta}\right\|_{(r)} \leqslant\left\|D^{s} x\right\|_{(r)}+\text { const }_{x}\left(|\Delta| /\left|\Delta t_{r}\right|\right)^{k} .
$$

Proof. By (7), $\left\|\varphi_{\Delta}-\varphi\right\|_{\infty}=O\left(|\Delta|^{k}\right)$, hence

$$
\left\|D^{s}\left(x_{\Delta}-x\right)\right\|_{\infty}=O\left(|\Delta|^{k}\right) \text { for } s=0, \ldots, m,
$$

which proves a stronger inequality than (10) for $s=0, \ldots, m$. For $s>m$ one now proceeds as in the proof of Lemma 4.1 in de Boor and Swartz [2] (with the role of $x$ and $R x$ played here by $x$ and $x_{\Delta}$, respectively) to show, using (11), that

$$
\left\|D^{s}\left(x-x_{\Delta}\right)\right\|_{(r)} \leqslant \text { const }_{x}\left(|\Delta| /\left|\Delta t_{r}\right|\right)^{k} \text { for } s=m+1, \ldots, m+k,
$$

in case $x \in \mathrm{L}_{\infty}^{(m+k)}\left[t_{r}, t_{r+1}\right]$. But, by (4), $x=M^{-1} \varphi$ has even $m+k+n$ continuous derivatives.

THEOREM 3.1. Let $T$ be the compact linear map on $Y\left(=\mathbf{L}_{p}[0,1]\right.$ for some $1 \leqslant p \leqslant \infty$ ) given by (1.2), with $M$ and $N$ given by (0.2) and satisfying (4), let $\mu$ be a nonzero eigenvalue of $T$ with corresponding invariant subspace $S$, and let $J$ be a matrix representation for $\left.T\right|_{S}$. Let $T_{\Delta}$ be the collocation approximation (1.6) to $T$, and assume that the collocation points $\left(\rho_{i}\right)$ satisfy (3).

Then, for all small $|\Delta|, T_{\Delta}$ has an invariant subspace $S_{\Delta}$ so that some matrix representation $J_{\Delta}$ for $\left.T_{\Delta}\right|_{s_{\Delta}}$ satisfies

$$
\left\|J-J_{\Delta}\right\| \leqslant \text { const }|\Delta|^{n+k}
$$

for some $\Delta$-independent const. 
Proof. By Theorem 2.2, we only need to show that the integrals in (2) are $0 .\left(|\Delta|^{n+k}\right)$ under our assumptions. But, by Lemmas 3.1 and 3.2 and (5),

$$
\begin{aligned}
\left|\int_{0}^{1} \varphi_{i}^{*}\left(T-T_{\Delta}\right) \varphi_{j, \Delta}\right| \leqslant \sum_{r}\left|\int_{t_{r}}^{t_{r+1}} \varphi_{i}^{*}\left(T-T_{\Delta}\right) \varphi_{j, \Delta}\right| \\
\leqslant \sum_{r} \text { const }\left|\Delta t_{r}\right|^{n+k+1}\left\|\varphi_{i}^{*}\right\|_{n,(r)}\left\|\left(T-T_{\Delta}\right) \varphi_{\Delta, j}\right\|_{n+k,(r)} \\
\leqslant \sum_{r} \text { const }\left|\Delta t_{r}\right|^{n+k+1}\left\|\varphi_{i}^{*}\right\|_{n,(r)} \max _{j}\left(\left\|x_{j}\right\|_{m+k,(r)}+\operatorname{const}_{x_{j}}\left(|\Delta| / \Delta t_{r}\right)^{k}\right) \\
\leqslant \text { const }|\Delta|^{k+n} .
\end{aligned}
$$

For completeness, we summarize previous results concerning the approximation of eigenvalue problems $(0.1)$ by collocation. Winther [14] was the first to publish results for the collocation of $(0.1)$ using $\mathrm{P}_{k+m, \Delta}^{m+1}$. He proved that (the quantity he called "algebraic multiplicity" we have called "ascent")

$$
\begin{aligned}
& \left|\lambda-\lambda_{\Delta}\right|=O\left(|\Delta|^{k / \alpha}\right), \quad \alpha=\text { the ascent of } \lambda ; \\
& \left\|D^{i}\left(u-u_{\Delta}\right)\right\|_{\infty}=O\left(|\Delta|^{k}\right), \quad i \leqslant m \text { if } \alpha=1 .
\end{aligned}
$$

In one of his numerical experiments, he collocated at the $k$ Gauss points even though his theory no longer applied. But this experiment demonstrated that some sort of "superconvergence" might be going on for the approximate eigenvalues, although its character could not be estimated. In his thesis [15], Winther proved that, using $\mathbf{P}_{k+m, \Delta}^{m}$ instead and with $\hat{m}:=m-($ order of $N)$,

$$
\begin{array}{cc}
\left|\lambda-\lambda_{\Delta}\right|=O\left(|\Delta|^{(k+\min (n, \hat{m})) / \alpha}\right), & \alpha=\text { ascent of } \lambda ; \\
\left\|D^{i}\left(u-u_{\Delta}\right)\right\|_{\infty}=O\left(|\Delta|^{k+\min (n, \hat{m})}\right), & i \leqslant m-\hat{m} ; \\
\left\|D^{i}\left(u-u_{\Delta}\right)\right\|_{\infty}=O\left(|\Delta|^{k+\min (n, m-i)}\right), & m-\hat{m}<i \leqslant m-1 ;
\end{array}
$$

these last assuming that $\alpha=1$. Extensive numerical work reported there also indicated that some sort of superconvergence was taking place for the approximate eigenvalues, when Gauss points were used in the collocation. Lathrop [10] presented some more numerical evidence of superconvergence for two second order operators; but still the character of the superconvergence could not be estimated. Cerutti and Parter [6] contemplated collocating the selfadjoint second order eigenvalue problem $\left(D^{2} u\right)(x)=$ $\lambda a_{0}(x) u(x)$ with $k$ Gauss points using $\mathbf{P}_{k+2, \Delta}^{2}$. Convergence rates were not relevant to that paper; what is worth noting is their proof that the approximate eigenvalues of the nonselfadjoint matrix problem were, in fact, real.

In their monograph [7] concerned primarily with collocation, using piecewise polynomials, Douglas and Dupont steered clear of straightforward collocation for the eigenvalue problem. However, they did use Bramble and Osborn's precursor [5] to Osborn's basic paper [11] to prove that for the second order problem $M u=\lambda u$, and using $\mathbf{P}_{k+2, \Delta}^{2}$, one can collocate the orthogonal projection of the right-hand side onto $\mathbf{P}_{k, \Delta}$ at the Gauss points to obtain (they suppose that the ascent of $\lambda$ is one) 


$$
\begin{gathered}
\left|\lambda-\lambda_{\Delta}\right|=O\left(|\Delta|^{2 k}\right) ; \quad\left\|u-u_{\Delta}\right\|_{2}=O\left(|\Delta|^{k+2}\right) ; \\
\max _{j}\left|D^{i}\left(u-u_{\Delta}\right)\left(x_{j}\right)\right|=O\left(|\Delta|^{2 k}\right), \quad i=0,1 .
\end{gathered}
$$

From the start, our numerical experiments indicated quite clearly that straightforward collocation of (0.1) at the Gauss points using $\mathbf{P}_{k+m, \Delta}^{m}$ should yield (at least, when the ascent of $\lambda$ is one)

$$
\left|\lambda-\lambda_{\Delta}\right|=O\left(|\Delta|^{2 k}\right) ; \quad \max _{j}\left|D^{i}\left(u-u_{\Delta}\right)\left(x_{j}\right)\right|=O\left(|\Delta|^{2 k}\right), \quad i<m ;
$$

i.e., about the best one could hope for from the known results for the boundary value problem [2]. It was these experiments, of course, that prompted us to develop this paper which completes Winther's work.

4. In Collocation, the Adjoint Problem is Not Approximated All That Well. In this section, we elaborate on our earlier contention that Osborn's bound (2.8) is not strong enough to give the desired $O\left(|\Delta|^{2 k}\right)$ bound for the eigenvalue error in Gausspoint collocation. The difficulty lies with the term

$$
\left\|\left.\left(T^{*}-T_{\Delta}^{*}\right)\right|_{S *}\right\|
$$

which, so we claim, cannot be shown to be $O\left(|\Delta|^{k}\right)$ merely because $S^{*}$ is a finite dimensional linear space of smooth function(al)s. Throughout this section, we settle on $Y=\mathbf{L}_{2}[0,1]$.

We begin with a simple bound on $\left.\left(T^{*}-T_{\Delta}^{*}\right)\right|_{S^{*}}$ which has, in fact, nothing to do with $S^{*}$ but merely relies on the obvious fact that

$$
\left\|\left.\left(T^{*}-T_{\Delta}^{*}\right)\right|_{S^{*}}\right\| \leqslant\left\|T^{*}-T_{\Delta}^{*}\right\|=\left\|T-T_{\Delta}\right\| .
$$

For this, let $\hat{m}$ be the difference between the order $m$ of $M$ and the order of $N$.

LEMmA 4.1. Under the assumptions of Theorem 3.1,

$$
\left\|T-T_{\Delta}\right\|=O\left(|\Delta|^{\hat{m}}\right) \text {. }
$$

Proof. For $f \in \mathbf{L}_{2}$, we compute with (1.3) that

$$
\left(T-T_{\Delta}\right) f=\int_{0}^{1}\left(1-P_{\Delta}\right) T(\cdot, s) f(s) d s .
$$

Further, by (1.3) and because of the smoothness assumption (3.4), $T(\cdot, s) \in$ $\mathbf{L}_{\infty}^{(\hat{m})}[0,1]$ uniformly in $s$, i.e.,

$$
\left\|D^{\hat{m}} T(\cdot, s)\right\|_{\infty} \leqslant \text { const }_{M, N} \text { for } 0 \leqslant s \leqslant 1 .
$$

consequently, from (1.7),

$$
\left\|\left(1-P_{\Delta}\right) T(\cdot, s)\right\|_{\infty} \leqslant|\Delta|^{\hat{m}} \text { const }_{M, N}
$$

and (2) follows. On combining (2) and (1), we then obtain

$$
\left\|\left.\left(T^{*}-T_{\Delta}^{*}\right)\right|_{S^{*}}\right\|=O\left(|\Delta|^{\hat{m}}\right)
$$


which allows the conclusion from (2.8) that

$$
\left|\mu-\bar{\mu}_{\Delta}\right|=O\left(|\Delta|^{k+\hat{m}}\right),
$$

and also gives the results (3.12) of Winther [15].

Next, we show that (3) is sharp in the sense that we cannot obtain a rate better than $O\left(|\Delta|^{\hat{m}}\right)$, if all we know about $S^{*}$ is that it is spanned by finitely many smooth function(al)s. For this, we exhibit a simple $M, N,\left(\beta_{i}\right)_{0}^{m-1}, \Delta$ and a very smooth $\varphi^{*}$ for which

$$
\left\|\left(T^{*}-T_{\Delta}^{*}\right) \varphi^{*}\right\|=\text { const }|\Delta|^{\hat{m}}
$$

We take $\varphi^{*}=1$, i.e.,

$$
\varphi^{*} f=\int_{0}^{1} f(t) d t, \quad \text { all } f \in \mathbf{L}_{2} .
$$

Then

$$
\begin{aligned}
{\left[\left(T^{*}-T_{\Delta}^{*}\right) \varphi^{*}\right] f } & =\int_{0}^{1} \varphi^{*}(t) \int_{0}^{1}\left(1-P_{\Delta}\right) T(t, s) f(s) d s d t \\
& =\int_{0}^{1}\left[\int_{0}^{1}\left(1-P_{\Delta}\right) T(t, s)\right] d t f(s) d s
\end{aligned}
$$

and, consequently,

with

$$
\left\|\left(T^{*}-T_{\Delta}^{*}\right) \varphi^{*}\right\|=\left\|\int_{0}^{1} e(t, \cdot) d t\right\|_{2}
$$

$$
e(\cdot, s):=\left(1-P_{\Delta}\right) T(\cdot, s)
$$

Next, we choose $M=D^{m}, N=D^{m-\hat{m}}$ and any appropriate $\left(\beta_{i}\right)_{1}^{m}$ independent over $\mathbf{P}_{m}$. Then, for some appropriate $p_{s} \in \mathbf{P}_{m}$,

$$
T(t, s)=N_{t}\left[(t-s)_{+}^{m-1} /(m-1) !-p_{s}(t)\right]=(t-s)_{+}^{\hat{m}-1} /(\hat{m}-1) !-q_{s}(t)
$$

for some appropriate $q_{s} \in \mathbf{P}_{\hat{m}}$. Also, $P_{\Delta}$ is then just $Q_{\Delta}$, i.e., interpolation from $\mathbf{P}_{k, \Delta}$ at the collocation points $\left(\tau_{i}\right)_{1}^{k l}$. Therefore, with the assumption that $k \geqslant m$,

$$
e(\cdot, s)=\left(1-Q_{\Delta}\right) T_{0}(\cdot, s)
$$

where

$$
T_{0}(\cdot, s):=(\cdot-s)_{+}^{m-1} /(\hat{m}-1) !
$$

Further, $e(\cdot, s)=\left(1-Q_{\Delta}\right) T_{0}(\cdot, s)$ vanishes on every interval $\left(t_{i}, t_{i+1}\right)$, which does not contain $s$ in its interior. Thus

$$
\int_{0}^{1} e(t, s) d t=\int_{t_{i}}^{t_{i+1}} e(t, s) d t
$$

with $i$ chosen so that $\left[t_{i}, t_{i+1}\right]$ contains $s$. On this interval, we change variables, using

$$
t=\sigma_{i}(\tau):=\left(t_{i}+t_{i+1}+\tau \Delta_{i}\right) / 2
$$


Then we can write

$$
\int_{t_{i}}^{t_{i+1}} e(t, s) d t=\int_{-1}^{1}(1-Q) T_{0}\left(\sigma_{i}(\tau), s\right) \frac{\Delta t_{i}}{2} d \tau,
$$

with $Q$ polynomial interpolation to functions of $\tau$ at the points $\rho_{1}<\cdots<\rho_{k}$, all in $(-1,1)$. Since also, with $\sigma:=\sigma_{i}^{-1}(s)$,

$$
T_{0}\left(\sigma_{i}(\tau), s\right)=\left(\frac{\Delta t_{i}}{2}\right)^{\hat{m}-1}(\tau-\sigma)_{+}^{\hat{m}-1} /(\hat{m}-1) !
$$

we therefore find that

$$
\int_{0}^{1} e(t, s) d t=\left(\frac{\Delta t_{i}}{2}\right)^{\hat{m}} /(\hat{m}-1) ! \cdot F(\sigma), \quad \sigma=\sigma_{i}^{-1}(s), \quad t_{i} \leqslant s \leqslant t_{i+1},
$$

where $F$ is the function on $[-1,1]$ given by

$$
F(\sigma):=\int_{-1}^{1}(1-Q)(\tau-\sigma)_{+}^{\hat{m}-1} d \tau
$$

Note that $\|F\|_{2}>0$ since, e.g., $Q(\cdot-\sigma)_{+}^{\hat{m}-1}=0$ for $\sigma>\rho_{k}$, hence

$$
F(\sigma)=\int_{-1}^{1}(\tau-\sigma)_{+}^{m-1} d \tau=(1-\sigma)^{\hat{m}} / \hat{m}>0 \text { for } \rho_{k}<\sigma<1 .
$$

Now combine (5) and (6) to get that

$$
\left\|\left(T^{*}-T_{\Delta}^{*}\right) \varphi^{*}\right\|^{2}=\left(\frac{\|F\|_{2}}{(\hat{m}-1) !}\right)^{2} \sum_{i=0}^{L-1}\left(\Delta t_{i} / 2\right)^{2 \hat{m}+1}
$$

For a uniform partition $\Delta=(i|\Delta|)_{0}^{l}$, therefore,

$$
\left\|\left(T^{*}-T_{\Delta}^{*}\right) \varphi^{*}\right\|=\frac{\|F\|_{2}}{(\hat{m}-1) ! 2^{\hat{m}+1 / 2}}|\Delta|^{\hat{m}},
$$

which proves our claim.

Note that the argument just given is local, and hence shows that $\left\|\left(T^{*}-T_{\Delta}^{*}\right) \varphi^{*}\right\|$ $\neq o\left(|\Delta|^{\hat{m}}\right)$ for any fixed smooth $\varphi^{*} \neq 0$ since such $\varphi^{*}$ is locally close to a constant.

Acknowledgement. We thank the referee for pointing out to us that a recent paper of Descloux, Nassif and Rappaz (RAIRO Anal. Numér., v. 12, 1978, pp. 113-119] contains a simplified proof of Osborn's results as well as observations like (2.13) and (2.14).

\section{Mathematics Research Center 610 Walnut Street \\ Madison, Wisconsin 53706}

T-7 Los Alamos Scientific Laboratory

P.O. Box 1663

Los Alamos, New Mexico 87545

1. K. AT KINSON, "Convergence rates for approximate eigenvalues of compact integral operators," SIAM J. Numer. A nal, v. 12, 1975, pp. 213-221.

2. C. DE BOOR \& B. SWARTZ, "Collocation at Gaussian points," SIAM J. Numer. Anal., v. 10, 1973, pp. 582-606. 
3. C. DE BOOR \& B. SWARTZ, "Collocation approximation to eigenvalues of an ordinary differential equation: Numerical illustrations." (Submitted to Math. Comp.)

4. C. DE BOOR \& B. SWARTZ, "Local piecewise polynomial projection methods for an ODE which give high-order convergence at knots." (Submitted to Math. Comp.)

5. J. H. BRAMBLE \& J. E. OSBORN, "Rate of convergence estimates for nonselfadjoint eigenvalue approximations," Math. Comp., v. 27, 1973, pp. 525-549.

6. JOHN H. CERUTTI \& S. V. PARTER, "Collocation methods for parabolic partial differential equations in one space dimension," Numer. Math., v. 26, 1976, pp. 227-254.

7. J. DOUGLAS, JR. \& T. DUPONT, Collocation Methods for Parabolic Equations in a Single Space Variable, Lecture Notes in Math., Vol. 385, Springer-Verlag, New York, 1974.

8. T. KATO, Perturbation Theory for Linear Operators, Springer-Verlag, Berlin and New York, 1966.

9. H.-O. KREISS, "Difference approximations for boundary and eigenvalue problems for ordinary differential equations," Math. Comp., v. 26, 1972, pp. 605-624.

10. JAMES F. LATHROP, "Using B-splines to solve the one-dimensional Schrödinger equation," J. Comput. Phys. (Submitted.)

11. JOHN E. OSBORN, “Spectral approximation for compact operators," Math. Comp., v. 29, 1975, pp. $712-725$.

12. G. M. VAINIKKO, "Rapidity of convergence of approximation methods in the eigenvalue problem," Ž. Vyčish. Mat. $i$ Mat. Fiz., v. 7, 1967, pp. 977-987= U.S.S.R. Computational Math. and Math. Phys., v. 7, 1967, pp. 18-32.

13. J. H. WIL KINSON, The Algebraic Eigenvalue Problem, Clarendon Press, Oxford, 1965.

14. R. WINTHER, "A collocation method for eigenvalue problems," $B I T$, v. 14, 1974, pp. 96-105.

15. R. WINTHER, En Kollokasjonsmetode for Egenverdiproblemer, Thesis for the Cand. Real Degree, University of Oslo, Norway, 1973.

16. K. A. WITTENBRINK, "High order projection methods of moment- and collocation-type for nonlinear boundary value problems," Computing, v. 11, 1973, pp. 255-274. 